\title{
Set-point Revisiting Of Maximum Impulse Protection Adjustment For DC Electric Traction Network
}

\author{
S.M. Kuznetsov \\ Novosibirsk State Technical University \\ Novosibirsk, Russian Federation \\ ksmetk@mail.ru \\ E.G. Langeman \\ Novosibirsk State Technical University \\ Novosibirsk, Russian Federation \\ langeman.evelina@gmail.com
}

\author{
B.V. Malozyomov \\ Novosibirsk State Technical University \\ Novosibirsk, Russian Federation \\ mbv5@mail.ru \\ A.A. Shtang \\ Novosibirsk State Technical University \\ Novosibirsk, Russian Federation \\ shtang@corp.nstu.ru
}

\begin{abstract}
The article outlines the transition from analogue to digital protection for DC traction network, selection methods of protection and their settings. The first trial uses results, suggestions for further enhancements of adjusting methods, using monitoring and increasing the level of automation and mathematical modeling.
\end{abstract}

Keywords-digital protection, method of protection adjustment, traction network, electric current.

\section{INTRODUCTION}

When the system operates (traction substation (TS), traction network $(\mathrm{TN})$, electric stock $(\mathrm{ES}))$, there are often failures of its individual elements caused by external influences, personnel mistakes, elements' wear, which are accompanied by short-circuits (SC). SC causes current leakage, which has the value of $20-50 \mathrm{kA}$ near TS of DC railways, and $2-6 \mathrm{kA}$ in the remote point substation near the neighboring TS bus. For underground and urban electric transport (UET), SC currents near TS are 6-20 kA, and in the remote point - 0.6-2 kA. In this case, the long-term permissible load currents may be commensurate with a minimum current of SC. Protection prevents the effects of SC; the system needs to disable the damaged item in hundredths of a second. Protection is the most widespread kind of automatics, which determines the possibility of the system of TS-TN-ES functioning and providing electricity supply reliability. Protection is always a standby mode waiting for a request for operation, which occurs in case of exceeding the controlled parameters of specified values, called as a set.

\section{THEORY}

Nowadays current protection based on determining the current in the circuit is mainly used. Current protection is divided into the maximum current protection (MCP) and the current cutoff (CC). If in the function of a current sensor there are differential relay type DRB, DNC or a built-in high-speed switch mechanism, such as a speed rise sensor (SRS), which responds to smooth or ramp current variation in a different way, it is called maximum impulse protection (MIP). All devices of electrical transport are now equipped with MIP. The difference between these protections is the way to provide selectivity (practically in a choice setting) that will be discussed later.

Thus, MIP is such kind of protection, which responds to value $I$ and current increment $\Delta I$ (current step). Current step $\Delta I$ is the difference between two adjacent steady-state values for the circuit current. For example, if current was changed (Figure. 1), $I_{0}$ and $I_{1}$ are established currents before and after a transient process and $\Delta I$ is a current step. Sensitivity analysis of MIP is usually made for the following transient modes: train's start, remote short-circuits, passage of blocked section insulator, switch of a section insulator on residual load. For a complete description of exponential or similar current step, constant current time $T_{1}$ must be specified and determined, as shown in Figure 1a.

But in case of pantograph separation or arcing, the current curve will have a few short steps with $T_{1}<T_{e}$ (Fig. 1b). The relay will interpret it as equivalent step $\Delta I_{e}$ with time constant $T_{E}$ that does not agree with the actuation calculated characteristics (Fig. 3) and false cutoff or protection failure will happened.

Let us consider a switch equivalent circuit with relay RMT, equipped with inductive shunt or relay DRB and protected feeder zone (Fig. 3a). In real traction networks $L_{1}>>L_{R}, L_{1}>>L_{s h}, R_{1}>>R_{R}, R_{1}>>R_{s h}$, so it may be assumed that switch settings $L_{R}, L_{s h}, R_{R}, R_{s h}$ will not 
affect current $I_{1}$ in external circuit $L_{1}, R_{1}$. For these initial conditions, it is possible to make the system of equations:

$$
\left.\begin{array}{l}
i_{1}=i_{\mathrm{p}}+i_{\mathrm{sh}} \\
i_{\mathrm{p}} R_{\mathrm{p}}+L_{p} \frac{d i_{\mathrm{p}}}{d t}=i_{\mathrm{sh}} R_{\mathrm{sh}}+L_{\mathrm{sh}} \frac{d i_{\mathrm{sh}}}{d t}
\end{array}\right\}
$$
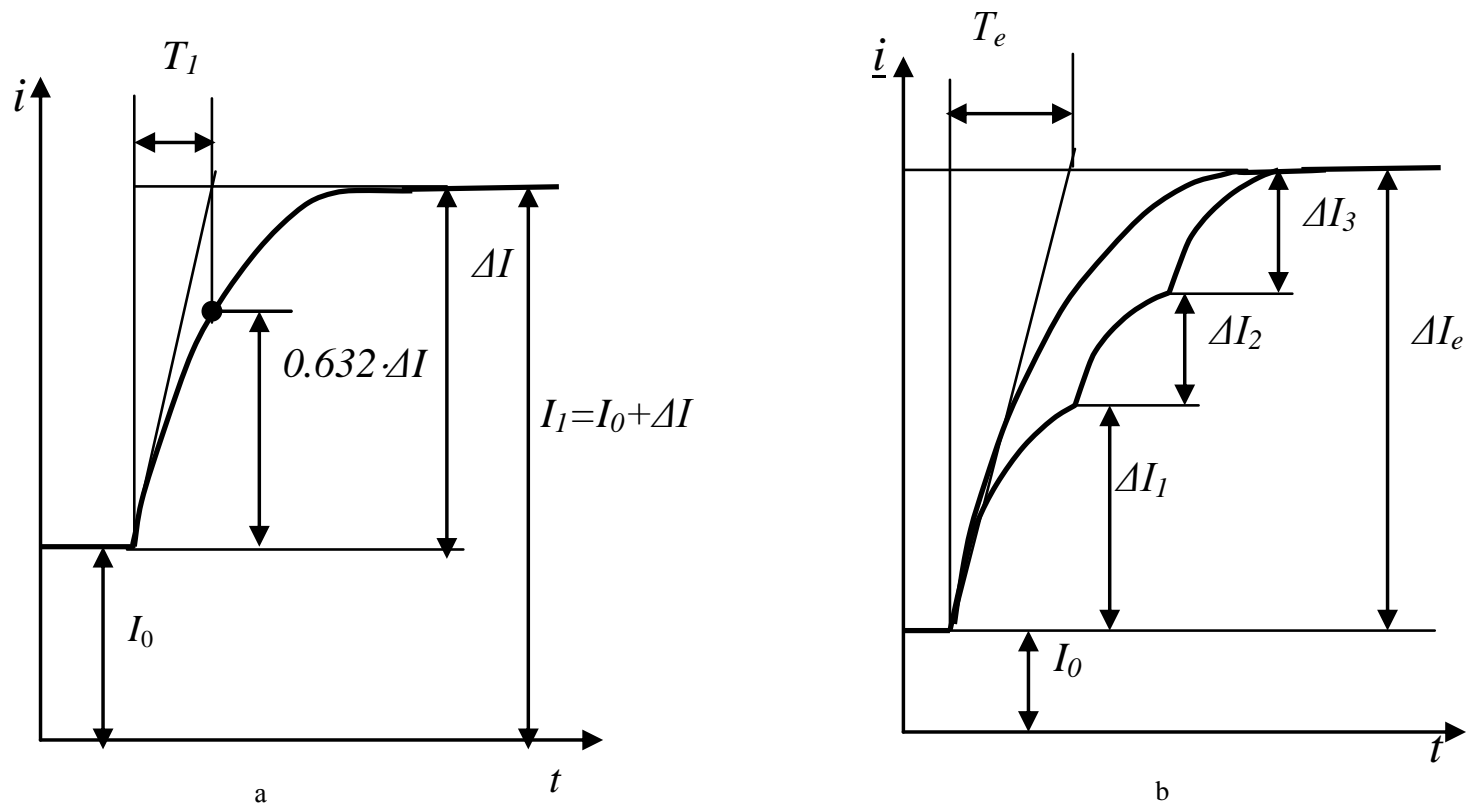

Fig. 1. Graph of current change from $I_{0}$ to $I_{1}$ explains the concept of current step $\Delta I$ and time constant $T_{1}$ of circuit during a single increment - a); continuous sequence of "steps" $\Delta \mathrm{I}_{1}, \Delta \mathrm{I}_{2}, \Delta \mathrm{I}_{3}$ and equivalent exponential step $\Delta \mathrm{I}_{\mathrm{e}}$ with the time constant $\mathrm{T}_{\mathrm{e}}-\mathrm{b}$ )

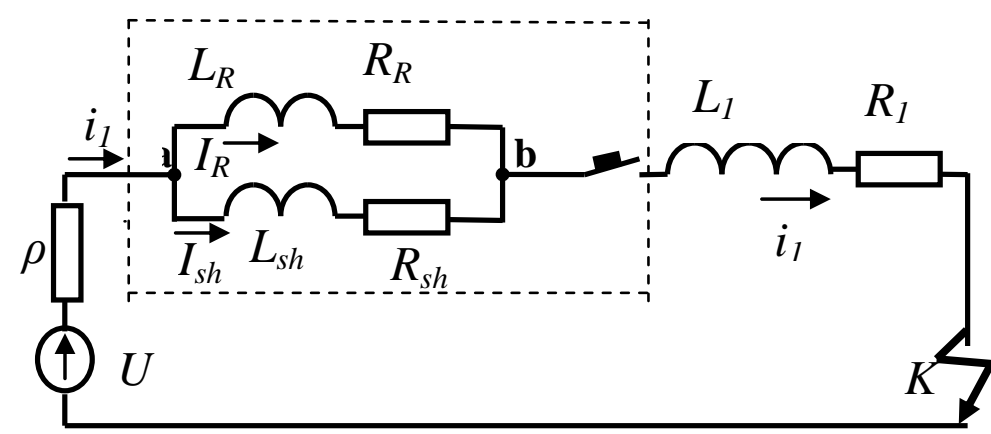

Fig. 2. The equivalent circuit high-speed breaker (HB) with inductive shunt: a) operation characteristic b) $R D S H$ and protected circuit

The value of current $\Delta \mathrm{I}$ in the circuit, which is necessary for relay operation (Fig. 2), depends on the current behavior. If it has a step with a small time constant, this difference is significantly less than a step with a very large time constant. This is because the most part of circuit current increment (step) will be held on chain $L_{p} \Rightarrow R_{p}$ (Fig. 3a, current $I_{R}$ ). This happens because the parallel circuit $L_{\mathrm{sh}} \Rightarrow R_{s h}$ has steel sheets packages with a large inductance, which slows down the changing of current $I_{s h}$.

The $I_{R}$ relay coil current cutoffs a switch. Using the lookup and derivation operations for equations (1), one will obtain:

$$
i_{\mathrm{p}} R_{\mathrm{p}}+L_{\mathrm{p}} \frac{d i_{\mathrm{p}}}{d t}=i_{1} R_{\mathrm{sh}}-i_{\mathrm{p}} R_{\mathrm{sh}}+L_{\mathrm{sh}} \frac{d i_{1}}{d t}-L_{\mathrm{sh}} \frac{d i_{\mathrm{p}}}{d t}
$$




$$
\frac{d i_{\mathrm{p}}}{d t}+i_{\mathrm{p}} \frac{\beta+1}{(\alpha+1) T_{\mathrm{sh}}}=\frac{d i_{1}}{d t} \frac{1}{(1+\alpha)}+i_{1} \frac{1}{(1+\alpha) T_{\mathrm{sh}}}
$$

This equation defines the law of current change in the cutoff relay coil in function of $i_{1}$ in the protected line. From equation (3), an expression for current $I_{R}$, which is maximum current $I_{R \max }$ in the cutoff coil could be obtained. With the certain ratios of inductive shunt parameters and demagnetization coil, when $L_{R} / L_{s h}=R_{R} / R_{s h}, \alpha=\beta$, current $I_{R}$ varies in proportion to current $I_{1}$ changing in the protected circuit. Therefore, such protection responds only to the current change. The maximum value of the current $I_{\max }$ in the circuit will reach at the end of the transition process $(t=\infty)$, then the current in cutoff coil is:

$$
i_{\mathrm{p} \max }=\left(I_{0}+\Delta \mathrm{I}\right) \frac{R_{\mathrm{sh}}}{R_{\mathrm{P}}+R_{\mathrm{sh}}}=k_{\mathrm{st}} I_{\max }
$$

where $k_{\mathrm{st}}$ - static coefficient, which is equal $k_{\mathrm{st}}=R_{\mathrm{sh}} /\left(R_{\mathrm{p}}+R_{\mathrm{sh}}\right)$ and shows which part of the current circuit branches in cutoff coil with its smooth variation, when $L_{\mathrm{sh}}=L_{\mathrm{p}}=0$. If $k_{\mathrm{st}} \leq 1$, then it is a usual maximum current protection (MCP).

If the parameters of inductive shunt were chosen, so that $L_{\mathrm{p}} / L_{\mathrm{sh}}$ has bigger value that $R_{\mathrm{p}} / R_{\mathrm{sh}} \cdot \alpha>\beta$, differentiate the expression for current and equating it to zero, we can find the time $t_{\max }$ to reach the current $i_{\mathrm{p}}$ maximum value.

$$
i_{\mathrm{p} \max }=\left(I_{0}+\Delta I k_{\mathrm{d}}\right) K_{\mathrm{st}}
$$

where $\mathrm{k}_{\mathrm{D}}$ - dynamic coefficient of MIP:

$$
k_{\mathrm{D}}=1+\frac{T_{\mathrm{sh}}(\alpha-\beta)}{(1+\alpha) T_{\mathrm{sh}}-(1+\beta) T_{1}} \exp \left(-\frac{t(1+\beta)}{(1+\alpha) T_{\mathrm{sh}}}\right)-\frac{(1+\beta)\left(T_{\mathrm{sh}}-T_{1}\right)}{(1+\alpha) T_{\mathrm{shu}}-(1+\beta) T_{1}} \exp (-
$$

where $\alpha=R_{\mathrm{p}} / R_{\mathrm{sh}}$ and $\beta=L_{\mathrm{p}} / L_{\mathrm{sh}}$ - parameters of cut-off switch or DRB, $T_{\mathrm{sh}}=L_{\mathrm{sh}} / R_{\mathrm{sh}}$ - shunt time constant, $T_{\mathrm{p}}=L_{\mathrm{p}} / R_{\mathrm{p}}$ - demagnetization coil time constant, $R_{1}$ protected circuit time constant. As we can see from (6) dynamic coefficient depends on the settings of the cut-off switch and protected lines not linearly, while [1-3] recommended for it hard values: for $\mathrm{HB}-1.05$, for relay DRB1.15. Thus, the possibility of either false operations or failures are taken into account.

It is obvious that protection operates when the cutoff coil current $I_{R \max }$ (Fig. 2a) has larger value than specified current of regulating point $I_{R U}, I_{R \max }>I_{R U}$ and does not operate if $I_{R \max }<I_{R U}$. Operating boundary could be defined from the condition:

$$
I_{R \max }=I_{R U}
$$

To construct protection operating characteristic parameter, values from (5) and (6) should be used in expression (7). Supposing that in operation, moment current is equal to line load $I_{0}=I_{\text {Oav }}^{\prime}$, it means that current is equal to static regulating point, $\Delta I=\Delta I_{a v}^{\prime}-$ is a dynamic component of operating current, that is a value of a current step, which cutoffs in presence of $\mathrm{I}_{0 \mathrm{av}}$. We shall obtain the equation for current impulse protection's (CIP) operation characteristic while $\mathrm{T} 1=$ const. Based on equation (8), cutoff switch operation characteristic could be constructed. Let us rewrite (8):

$$
\begin{gathered}
\Delta I_{\mathrm{av}}^{\prime}=\frac{i_{\mathrm{RU}}}{C}-\frac{I_{0 a v}}{B C}, \text { where } \\
C=\frac{1}{B}\left(1-e^{-\frac{B t_{\max }}{A T_{\mathrm{sh}}}}\right)+\frac{T_{\mathrm{sh}}-T_{1}}{B T_{1}-A T_{\mathrm{sh}}}\left(e^{-\frac{t_{\mathrm{max}}}{T_{1}}}-e^{-\frac{B t_{\max }}{A T_{\mathrm{sh}}}}\right), \\
A=\alpha+1, B=\beta+1 .
\end{gathered}
$$

Equation (8) is an intercept from for the equation of a straight line, where $\Delta \mathrm{I}^{\prime}$ av is a function:

$$
\Delta \mathrm{I}_{\mathrm{av}}^{\prime}=\Delta \mathrm{I}_{\mathrm{av}}\left(I_{0 \mathrm{av}}-I_{0 \mathrm{av}}^{\prime}\right) / I_{0 \mathrm{av}}^{\prime} \cdot \Delta \mathrm{I}_{\mathrm{av}}^{\prime}=\mathrm{f}\left(\mathrm{I}_{0 \mathrm{av}}^{\prime}\right) \text {, while } \mathrm{T}_{1}=
$$
const.

While $\mathrm{I}_{\text {Oav }}^{\prime}=0$ :

$$
\Delta I_{\mathrm{av}}^{\prime}=\Delta I_{\mathrm{av}}=\frac{i_{\mathrm{RU}}}{C}
$$

While $\Delta \mathrm{I}_{\mathrm{av}}^{\prime}=0$ :

$$
I_{0 \mathrm{av}}^{\prime}=I_{0 \mathrm{av}}=i_{\mathrm{RU}} B
$$

The values $I_{0 a v}$ and $\Delta I_{a v}$ are correspondingly static and dynamic regulating points of MIP, and dynamic regulating point depends on the current step time constant $T_{1}$.

The minimal value of $\mathrm{K}_{\mathrm{D}}$ (shunt amplification coefficient $k_{\mathrm{y}}$ [4]) corresponds to network time constant $T_{l}=0$, then:

$$
K_{\mathrm{d} \min }=\frac{A}{B}=\frac{(\alpha+1)}{(\beta+1)}=\frac{\left(L_{\mathrm{p}}+L_{\mathrm{sh}}\right) R_{\mathrm{sh}}}{\left(R_{\mathrm{p}}+R_{\mathrm{sh}}\right) L_{\mathrm{sh}}}
$$

For relay DRB:

$$
K_{d \min }=\frac{\left(4 L_{2}-L_{1}\right)\left(r_{1}-r_{2}\right)}{\left(r_{1}+r_{2}\right) L_{1}}
$$

While $T_{1} \geq T_{1 k p}=T_{\text {sh }}, \mathrm{t}_{\max }=\infty$, and static regulating point is equal to dynamic one; $\mathrm{K}_{\mathrm{D}}$ has a minimal value. In this case, $\mathrm{C}=1 / \mathrm{B}$, so $K_{\mathrm{d} \max }=1$ (Fig. 3). 


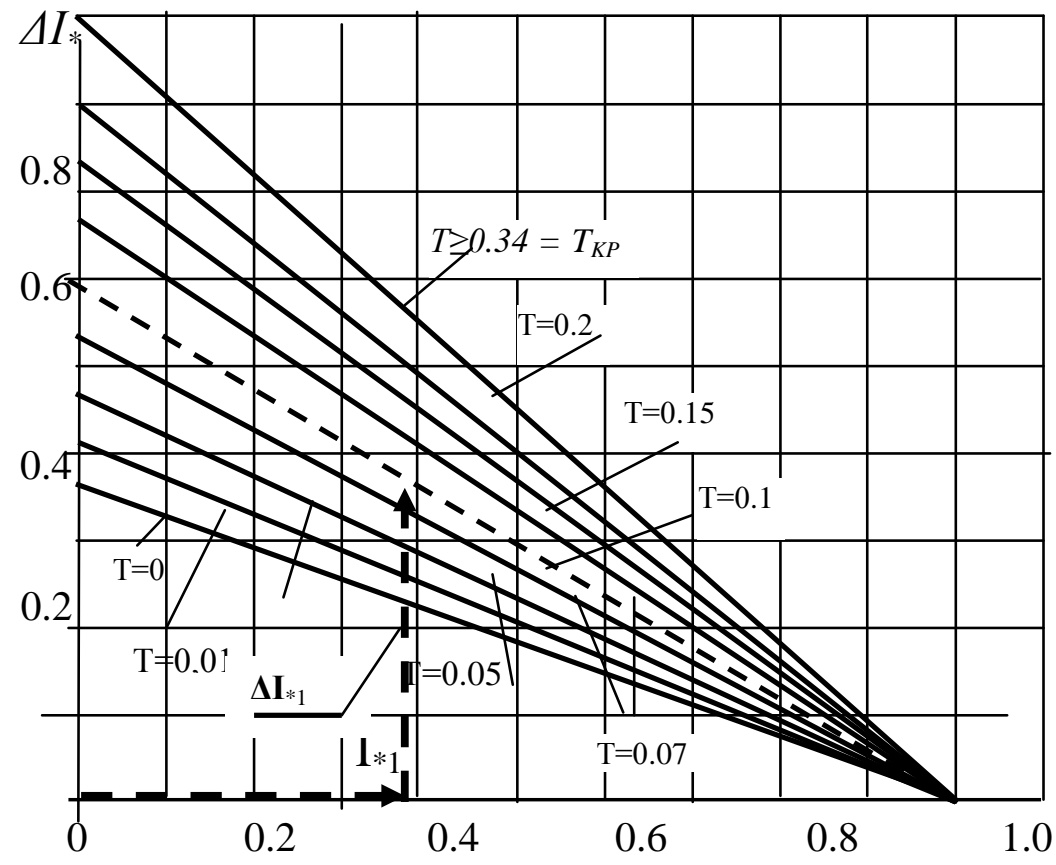

Fig.3. MIP set of curves $I_{a v}=f\left(I_{0}, \Delta I, T_{c}\right)$ for time constant $0<T_{C} \leq 0,34 \mathrm{~s}$; dashed line - checking procedure of MIP for operation with current $I_{* l,}$, step $\Delta I_{* l}$ for circuit with $T_{C}=0,1 \mathrm{~s}$ in relative units

This is the reason of frequent false operations, which happen during the exploitation [5-7].

To increase the reliability of protection operation, the following types of protection were offered at Novosibirsk State Technical University (NSTU) [8-10] (in part of protection determination). Calculation method of regulating point by means of mathematical modeling, feed current monitoring and traction network section protection verifying were also offered.

\section{EXPERIMENT}

The selection protection regulating point is based on the comparison of the normal mode parameters at maximum loads and steady short-circuit mode at the specified point. The regulatory requirements of protection sustainability against short-circuits are the basis for the selection. For selective protection, these conditions are following:

a) short-circuits sensitivity within the protected area (internal short-circuits);

b) distance from normal operation of protected bending;

c) distance from short-circuits, which are outside the protected area (external short-circuits).

The selecting method for regulating points for traditional protection based on the MIP includes the following main sections:

-analysis of section traction power network parameters;

-selection of calculation schemes for normal, forced and emergency regimes;

-creation of a database necessary for the calculation of load currents and short-circuit currents; -perform calculations using the program "Kortjes", or other analytical method with high precision;

-selection of types and areas of primary protection, identifying possible backup protection; calculation of protection settings, filling the regulating point card;

-examination of operation and protection availability of power network section.

The proposed methodology was tested on a real section of the railway.

\section{Operation reliability and protection availability of power network section examination.}

After carrying out the regulating point calculation, it is necessary to check the overlap of "dead" protection zones. The length of the catenary where protection device cannot selectively distinguish short-circuit currents from load currents is called a "dead" protection zone. In the same way, they include catenary sections where only one protection does not overlap the section length fully, for example, it overlaps only a half. "Dead" zones are in remote locations of catenary section.

Figure 4 shows the power layout of experimental section, section of MIP operation installed on each TS switch and sectioning point (SP).

In case of normal power layout SP-8, SP-23, SP-26 overlap "dead" zones of cutoff switches TS-250, TS-252, TS241. For example, $l_{d 3}$ has "dead" zone for $H B_{3}$ TS-252 and it is overlapped by protection $H B_{4 \mathrm{P}} \mathrm{SP}-8$. If switch $H B_{4 \mathrm{P}} \mathrm{SP}-8$ cutoffs because of $\mathrm{SC}$ at that point, there will be emergency mode not sensitive TS protection. Let us consider this case in more detail. When SC is in the zone of $l_{d 3}$ cutoff switch BV4P disconnects and power from TS-240 will cease. But near TS242 cutoff switch $\mathrm{HB}_{3}$ will remain operated, because 
protection in this area is not sensitive, and as a result SC in a remote point happens.

In protection section of traction network "dead" zone are different (Fig. 4). This is because the feed zones has different track profiles, brands of catenary wires and regulating point values of feed MIP. If regulating point is uprating, "dead"

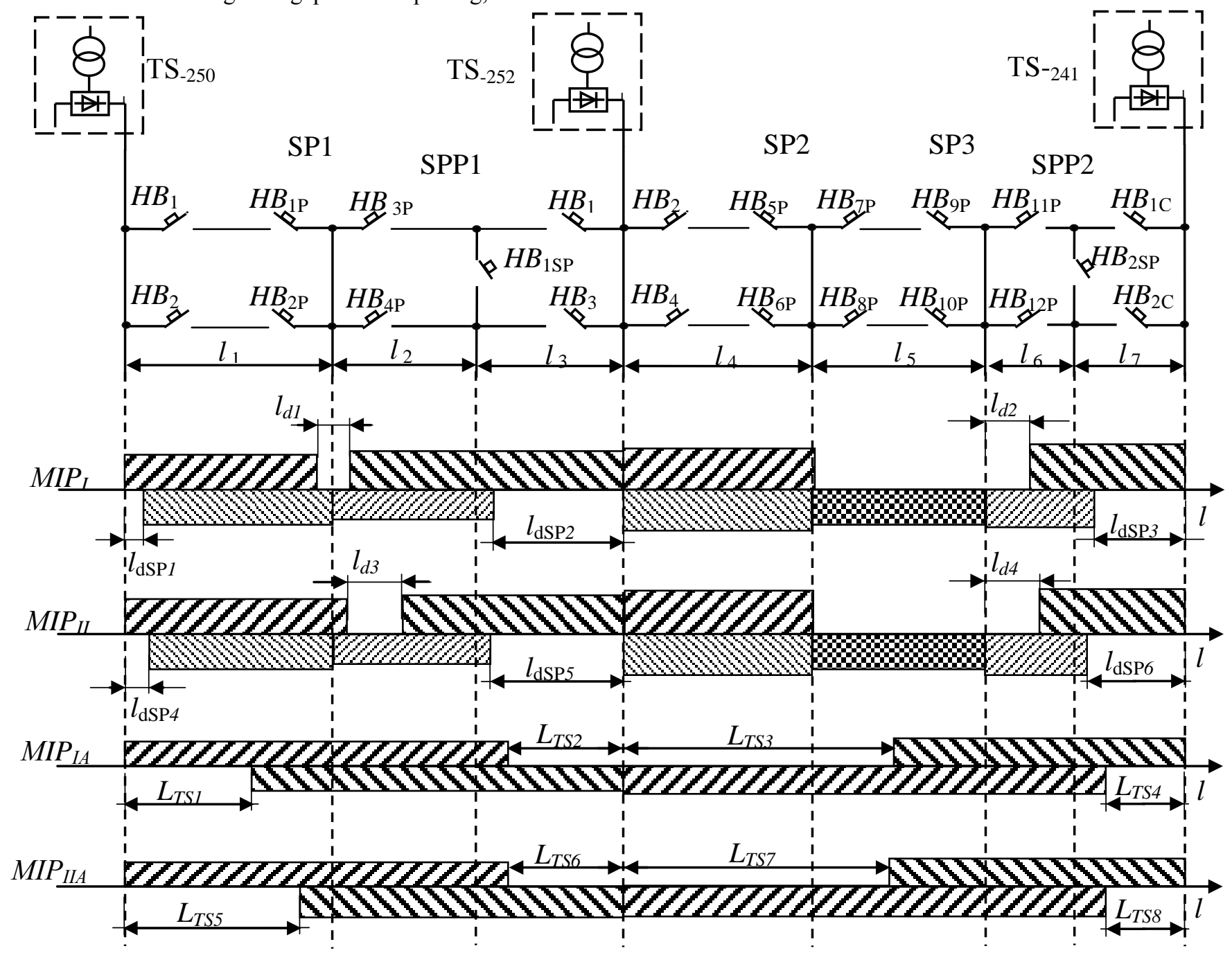

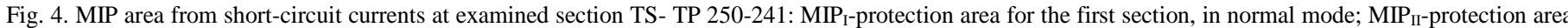
for the second section, in normal mode; $\mathrm{MIP}_{\mathrm{IA}}, \mathrm{MIP}_{\mathrm{IIA}}$-protection area for the first and second section, in forced mode; $l_{d l} \div l_{d 4}$-length of "dead" zones of protection in normal mode; $l_{d S P I} \div l_{d S P 6}$ - length of "dead" zones of sectioning points protection in normal mode, partitioning $l_{T S I} \div l_{T S 6}-$ length of "dead" zones of TS protection in a forced mode.

In the forced mode, sectioning points or parallel sectioning points (PSP) may be cutoff. Then the MIP operation area with regulating points for normal mode will not be able to block all of substation area. So the calculation for the emergency mode was made.

Lengths of MIP “dead" zones for TS cutoff switches in normal mode are: $l_{d 1}=1 \mathrm{~km} ; l_{d 2}=0,5 \mathrm{~km} ; l_{d 3}=2,3 \mathrm{~km} ; l_{d 4}=$ $0,5 \mathrm{~km}$. For ES cutoff switches: $l_{S P 1}=0,85 \mathrm{~km} ; l_{S P 2}=5,2 \mathrm{~km}$; $l_{S P 3}=5 \mathrm{~km} ; l_{S P 4}=0,82 \mathrm{~km} ; l_{S P 5}=5,4 \mathrm{~km} ; l_{S P 6}=5,2 \mathrm{~km}$. For TS cutoff switches in forced mode $l_{\mathrm{TS} l}=5,6 \mathrm{~km} ; l_{\mathrm{TS} 2}=4 \mathrm{~km}$; $l_{\mathrm{TS} 3}=4,6 \mathrm{~km} ; l_{\mathrm{TS} 4}=4 \mathrm{~km} ; l_{\mathrm{TS} 5}=7 \mathrm{~km} ; l_{\mathrm{TS} 6}=4 \mathrm{~km} ; l_{\mathrm{TS} 7}=4,1$ $\mathrm{km} ; l_{\mathrm{TS} 2}=3,7 \mathrm{~km}$. zone increases, protection sensitivity reduces. If regulating point is underrating, current loads become equal to SC currents, which reduces the protection selectivity and leads to an increase in false operations. 
system with an amplifying point", Power electronics and power engineering, vol. 9, pp. 8-18, 2014.

[2] O.I. Sablin, "Improving the efficiency of energy recovery in the electric transport system with limited traction power supply", Technological audit and production reserves, vol. 5/1, pp. 21-26, 2014.

[3] V.V. Biryukov, A.V. Kulekina, "The calculation features of the electrical energy storage devices parameters in transport", IFOST 2016, pp. 41-43, June 2016 [The 11 International forum on strategic technology, p. 596, 2016].

[4] V.I. Sopov, N.I. Schurov, Y.A. Prokushev and A.A. Shtang, "Increasing the efficiency of the use of electrical energy in the sub-subsystem of electric transport", Improvement of technical means of electric transport, vol. 3 (29), pp. 115-123, Jule 2002 [Digest of scientific works of the NSTU, p. 189, 2002].
[5] E.N. Bykov, B.V.Panin, V.N.Pupynin, Traction networks of subways, Moskow: Transport, 1987

[6] M.V. Tarnizhevsky, D.K. Tomlyakovich, Design of power supply devices for trams and trolleybuses, Moskow: Transport, 1986.

[7] K.G. Marquardt, Electrosupply of Electrified Railways, Moskow: Transport, 1982

[8] M.A. Sleptsov, Fundamentals of Electric Transport, Moskow: Academy, 2006.

[9] L.A. Bessonov, Theoretical bases of electrical engineering, Moscow: Higher School, 1973.

[10] V.I.Sopov, V.V. Biryukov, Y.A. Prokushev, Y.A. Rylov, "Analysis of power supply systems for rolling stock with various schemes of traction networks", Abstract journal of VINITI RAS: Transport, Science, Engineering, Management, vol. 2, pp. 49-53, 2008. 
